\title{
Shearing of granular materials in a confined split-bottom Couette cell
}

\author{
Mahnoush Madani ${ }^{1, *}$, Maniya Maleki ${ }^{1,2}$, and M. Reza Shaebani ${ }^{3}$ \\ ${ }^{1}$ Department of Physics, Institute for Advanced Studies in Basic Sciences, Zanjan 45137-66731, Iran \\ ${ }^{2}$ Optics Research Center, Institute for Advanced Studies in Basic Sciences, Zanjan 45137-66731, Iran \\ ${ }^{3}$ Department of Theoretical Physics \& Center for Biophysics, Saarland University, 66123 Saarbrücken, Germany
}

\begin{abstract}
Formation of shear bands is one of the most remarkable phenomena in the dynamics of granular matter. Several parameters have been so far identified to influence the behavior of the shear bands. We carried out experiments to investigate the evolution of the shear bands in the split-bottom Couette cell in the presence of confining pressure. We employed the Particle Image Velocimetry (PIV) to characterize the shear band both in the absence and presence of external pressure. Our results show that the location and width of the shear band are affected by both the confining pressure and the filling height. The shear zone evolves towards the middle of the cylinder and expands to a broader region with increasing applied pressure or filling height; also the angular velocity decreases relative to the rotation rate of the bottom disk. Our findings are consistent with prior empirical observations on the formation of wide shear bands at free surfaces.
\end{abstract}

\section{Introduction}

Granular matter is a collection of individual (macroscopic) solid particles, with a dissipative and athermal nature. As the applied shear stress on granular materials reaches a critical level, the material yields and flows differently from ordinary liquids. Since the constituent elements of a granular system are massive and have large sizes, they interact through frictional contacts and inelastic collisions when flowing, thus, the system continuously dissipates energy. A remarkable difference with ordinary flows is the localization of shear strain in relatively narrow zones, the socalled shear bands. Granular flows not only appear in everyday life but also in several industries, such as manufacturing, agriculture, food and pharmaceuticals. To store, transport and process primary materials such as ore, coal, sand, grains and all sorts of powders, one deals with shearing and flow of granular materials. These phenomena also play a major role, at macroscopic scales, in geophysical processes such as avalanches, landslides, sandstorms and earthquakes. Because of the important role of shear bands in geophysical events, numerous studies have explored their dynamic properties $[1,2]$.

One of the widely used experimental and simulation setups to study shear banding in dense and slowly flowing granular systems is the modified Couette cell [3-7]. While the original design of the Couette cell included two concentric cylinders where the inner one was rotating, in the modified Couette cell the inner part is replaced by a rotating disk at the bottom of the cell $[6,8]$. The advantage of this configuration is that it allows for the formation of a universal wide shear band away from the container walls $[6,7]$. This shear band is generated by the trans-

*e-mail: mahnoush.madani@iasbs.ac.ir

A video is available at https://doi.org/10.48448/21w2-6704 ferred shear to the bulk induced by the slow rotation of the split-bottom disk; thus, interparticle as well as particlewall friction coefficients are essential parameters in creating the wide shear band in the bulk of the system. In previous experimental studies, an empirical relation for the flow profile at the free surface was extracted. Also the dependence of the shear-band evolution on the ratio of the filling height $H$ to the radius $R_{C}$ of the bottom disk with a rough surface was studied $[6,7,9]$. The behavior of the granular flow at the free surface in terms of the radius $r$ from the cylinder axis was described as $\frac{\omega(r)}{\Omega}=\frac{1}{2}+\frac{1}{2} \operatorname{erf}\left[\frac{\left(r-R_{s}\right)}{\delta}\right]$, where $R_{S}$ is the location of the centre of the shear band, $\delta$ is its width, and $\Omega$ is the constant angular velocity of the bottom disk [7]. Also, it was shown that the location of shear band in the absence of external pressure follows $\frac{R_{c}-R_{s}}{R_{c}}=\left(\frac{H}{R_{c}}\right)^{\frac{5}{2}}$, while its width expands as $\delta \sim H^{\frac{2}{3}}$ [7]. Since the shear rate is low and particles mostly have frictional contacts, the system is in the slow transitional state, where the shear stress is independent of the magnitude of shear rate but depends on the stresses applied on the boundaries [2]. It has been shown that increasing the filling height $H$ causes axial slip; the dissipation due to shear transformation between different layers increases, resulting in the gradual decrease of angular velocity at the surface, as well as the deviation of the flow profile from the error function form $[4,9,10]$.

Here we aim at experimentally investigating the effect of confining external pressure $P$ on the flow profile and the characteristics of the shear band.

\section{Experimental setup}

Our experimental setup is a split-bottom Couette cell as shown in Figure 1, with the bottom split at $R_{c}=7.5 \mathrm{~cm}$, 


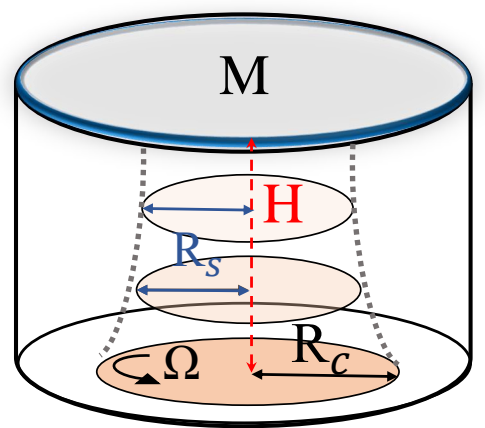

Figure 1. Schematic sketch of the experimental setup. In the split-bottom Couette cell, the bottom plate is divided into two parts: the stationary outer part $\left(r>R_{C}\right)$ and the rotating inner part (with the angular velocity $\Omega$ ). The Loading weight $M$ is a thick transparent plexiglass plate, placed on the top of the granular pile. Dotted lines show the location of shear band $R_{s}$ inside the bulk for shallow filling heights.

the outer cylinder radius $R=10 \mathrm{~cm}$, and a constant angular rotation $\Omega=0.13 \mathrm{rad} / \mathrm{s}$. In order to uniformly apply the external pressure and record the dynamics of the system from the top surface, we use disk-shaped loading weights $M$ made of transparent plexiglass with the same radius as the cell. These plates have different thicknesses and, therefore, various weights between 50 to 550 gr. Our granular material in the bulk of the system consists of glass beads with an average size of $2 \mathrm{~mm}$ and polydispersity of approximately $15 \%$. In our experiments, the cell is filled up to different heights between 0.5 to $4 \mathrm{~cm}$.

In order to illuminate the system, we used commercial fluorescent lamps and we used a Casio EX-F1 digital camera with pixel resolution of $2 \mu \mathrm{m}$ and a frame rate of 30 frames/second, fixed at the place where the camera axis is perpendicular to the surface of our granular system to capture particle flow evolution. We started recording after the granular flow reached the steady state. The recorded frames were processed and analysed by the Particle Image Velocimetry (PIV) method, to extract the surface velocity field of particles [11]. These velocity fields were transferred to polar coordinate with its center at the axis of the cell, which leads to the extraction of the temporal average of the angular velocity $\omega(r)$ at different radius $r$. This velocity profile can be used to extract the location and width of the shear band. Also, we measured the angular velocity near the cylinder axis to study the effects of the filling height and external pressure on the evolution of axial slip.

\section{Experimental Results}

The principal information that we directly extract from the PIV results is the angular velocity profile, which is partly presented in Figure 2. In each radius set, the error bars indicate the standard errors of the angular velocity. Apart from the area around the axis where velocity fluctuations are large due to low statistics, the deviation from the errorfunction form is relatively small over the rest of the surface, with the mean deviation being around $2.4 \%$ over all (a)
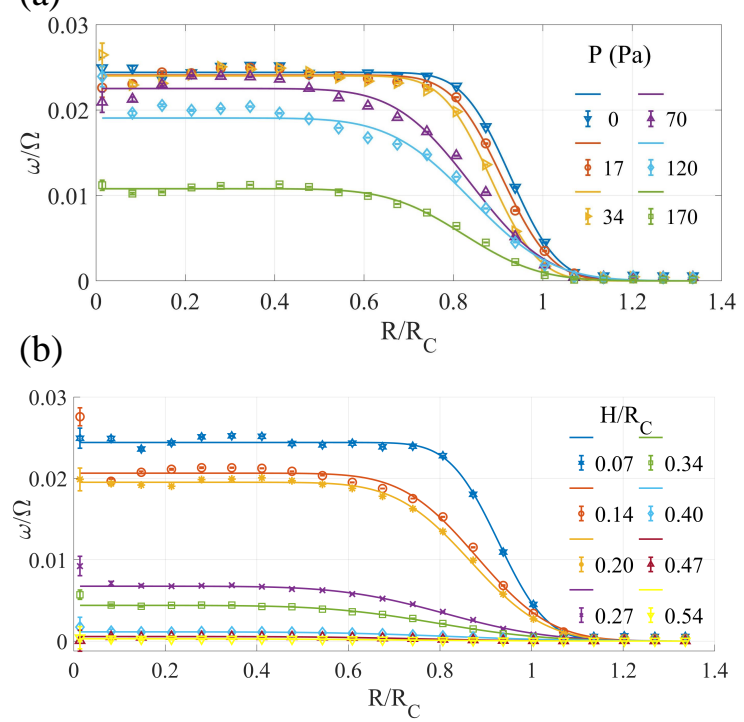

Figure 2. Shear band profile, $\omega(r) / \Omega$, versus normalised radius for (a) different pressures at the normalised filling height $H / R_{C}=0.07$, and (b) different filling heights under the constant external pressure $P=0$. The error bars in most cases are smaller than the symbols size.

data. The filling height ratio in Figure 2(a) is $H / R_{C}=0.07$, which is equal to the depth of half a centimetre of the granular material within the cell. In this figure, the first plot with $P=0$ indicates the free surface granular flow while the system is confined for $P>0$. With increasing the pressure, the shear band shifts towards the center of the cell and the angular velocity decreases in all radii. We also present the flow profile in the free surface case in Fig. 2(b) for different filling heights. The solid lines show the error function fitted to the experimental data. We observe similar trends with increasing filling height or external pressure. Indeed, the confining isotropic pressure affects the shear zone similarly to an additional column of grains that applies the same pressure on the surface.

A comparison between the panels (a) and (b) of Fig. 2 shows that the range of the applied pressure in our experiments is relatively weak compared to the weight of the granular column. For a quantitative comparison, we examine the reduction of the angular velocity at the center $\omega_{0}$ in terms of two variables: the external pressure $P$ and the filling height $H$. The central angular velocity $\omega_{0}$ can be deduced from $\omega_{r}(r)$ in the limit of $r \rightarrow 0$. In panel (a) of Figure 3, each set of the data presented with a distinct colour has a constant height, and the external pressure varies. By fitting an exponential function of the form $\omega_{0} / \Omega \propto \exp \left[-\alpha_{P} P\right]$ to the tail of each curve, we can extract the decay exponent $\alpha_{P}$, indicating the effect of increasing pressure on $\omega_{0}$, as shown in Figure 3(b). A similar analysis has been carried for the variation of height in constant pressure using $\omega_{0} / \Omega \propto \exp \left[-\alpha_{H} H / R_{c}\right]$. The decay exponent $\alpha_{H}$ is presented in Figure 3(d). In both plots of Figure 3(a) and (c), $\omega_{0}$ is displayed in logarithmic scales. Comparing the effect of increasing the filling 

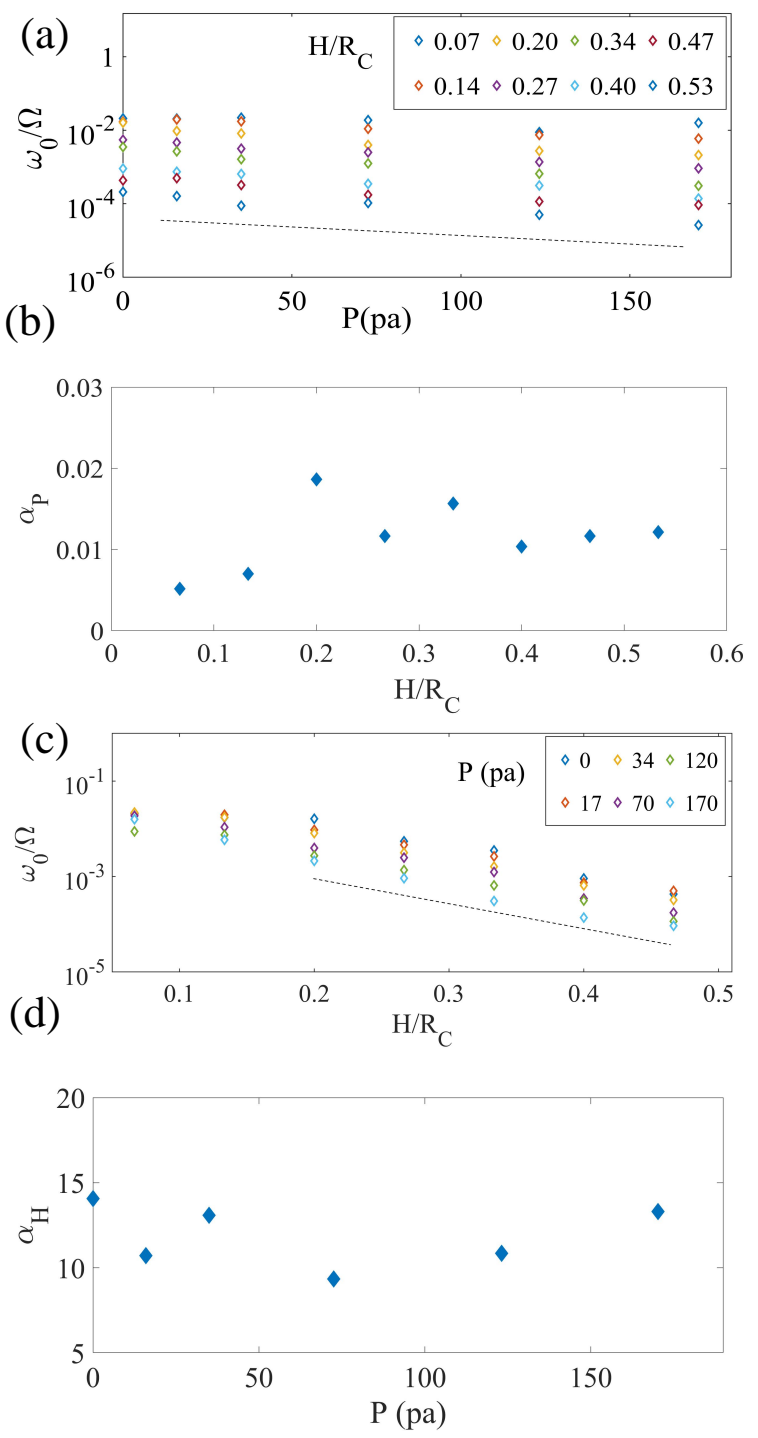

Figure 3. (a) $\omega_{0} / \Omega$ in term of $P$ for increasing values of the filling height $H / R_{c}$ from 0.07 to 0.53 . (b) The decay exponent $\alpha_{P}$ of the tail of $\omega_{0}-P$ curves at different values of $H$. (c) $\omega_{0} / \Omega$ in term of $H / R_{c}$ for increasing values of the external pressure $P$ from 0 to $170 \mathrm{pa}$. (d) The decay exponent $\alpha_{H}$ of the tail of $\omega_{0}-H$ curves at different values of $P$. The dotted lines indicate an exponential decay as a guide to the eye.

height of the granular column with increasing the external pressure in Figure 3, one can realise that while in panels (a) and (c) these two variables induce similar changes in the axial angular velocity $\omega_{0}$, the influence of the filling height is quantitatively stronger than the applied pressure in reducing the magnitude of the angular velocity. This is also reflected in the decay exponents $\alpha_{H}$ and $\alpha_{P}$. While these two quantities show no systematic dependence on their corresponding variables, the average amount of $\alpha_{H}$ is two orders of magnitude larger than $\alpha_{P}$, indicating that increasing the filling height in our setup reduces the angular velocity more dramatically than increasing the pressure within the studied range.

The evolution of the shear-band position and width with $P$ or $H$ can be understood by means of energy dissi-
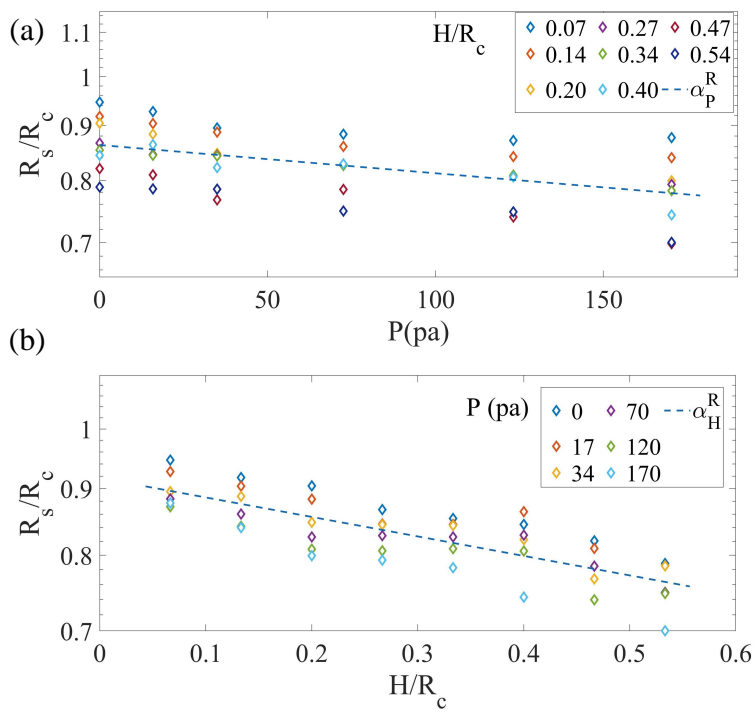

Figure 4. Position of the shear band, $R_{s} / R_{c}$, in terms of (a) $P$ for increasing values of the filling height $H / R_{c}$ from 0.07 to 0.54 (the dashed lines shows $\alpha_{P}^{R}$, the exponential decay of the average $R_{s} / R_{c}$ for increasing $P$ ) and (b) $H / R_{c}$ for increasing values of the external pressure $P$ from 0 to $170 \mathrm{pa}$ (the dashed lines shows $\alpha_{H}^{R}$, the exponential decay of the average $R_{s} / R_{c}$ for increasing $H$ ).

pation considerations, assuming that the shear band forms along a path in the bulk that minimizes the energy dissipation rate [10]. We compare a granular pile of total height $H$ with a free surface to a pile with height $H^{\prime}\left(H^{\prime}<H\right)$ under a confining pressure $P$. Considering the case that the extra pressure due to the difference between the filling heights balances the applied pressure (i.e. $\rho g\left(H-H^{\prime}\right)=P$ ), the bulk pressure at any filling height $0<h<H^{\prime}$ is the same in both systems, $p(h)=\rho g(H-h)=\rho g\left(H^{\prime}-h\right)+P$. To obtain the overall shape of the shear zone, the dissipation rate $\sigma v \mathrm{~d} a$ for a ring of sliding area $\mathrm{d} a$ should be integrated over the entire surface of the shear band and minimized. Here, $\sigma$ is the shear stress, which can be approximated as the isotropic pressure due to the agitation-induced fluidization of the system. Thus, $H$ and $P$ play a similar role in determining the path of the shear band at a given height in the bulk of the two systems. However, $H$ additionally contributes in determining the overall path as it sets the integral limit in the variational procedure [10]. As a result, the surface profiles of the two systems are different, while the bulk profile of the higher pile at $h=H^{\prime}$ is the same as the surface profile of the pile under confining pressure.

We also investigate the behavior of the location and width of the shear band. The location of the shear band is defined as the position where the shear rate $\dot{\gamma}$ is maximum. The shear rate can be derived from the angular velocity as $\dot{\gamma}=r \frac{d \omega}{d r}$. This quantity in the limit of narrow shear bands is equal to the value of $R_{s}$ that is extracted from the error function fit to the flow profile. Figure 4 demonstrates the $R_{S} / R_{c}$ under the variation of $P$ and $H / R_{c}$, in plots (a) and (b), respectively. In each plot, there is a dashed line, showing the exponential decay of the average behavior of all the presented data sets. The decay exponent value for increas- 

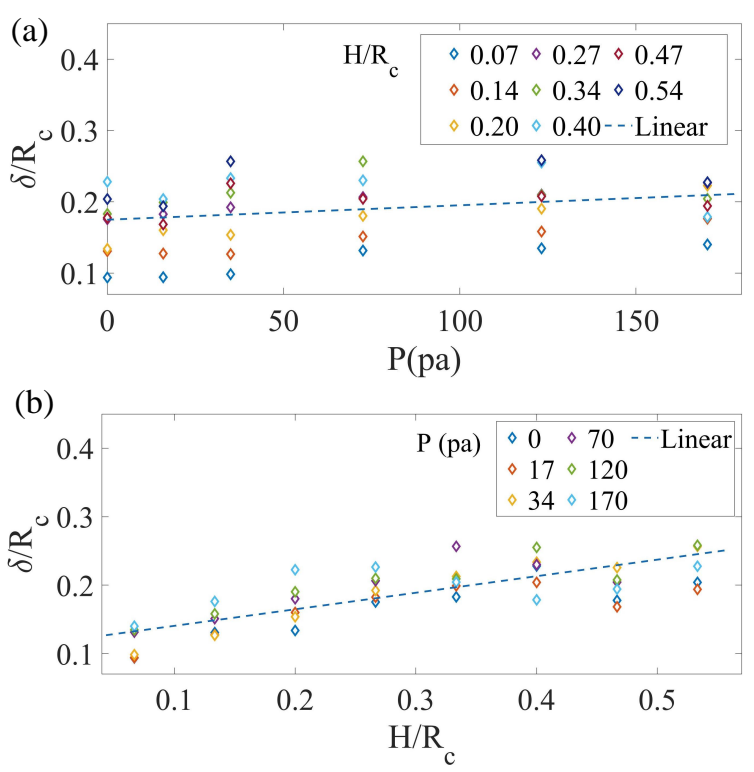

Figure 5. Width of the shear band, $\delta / R_{c}$, as a function of (a) $P$ for increasing values of the filling height $H / R_{c}$ from 0.07 to 0.54 (the dashed lines shows the linear fit to the average behavior of $\delta / R_{c}$ with increasing $P$ ) and (b) $H / R_{c}$ for increasing values of the external pressure $P$ from 0 to $170 \mathrm{pa}$ (the dashed lines shows the linear fit to the average behavior of $R_{s} / R_{c}$ with increasing $H$ ).

ing the external pressure is $\alpha_{P}^{R}=6 \times 10^{-4}$ while this value for rising the filling height is $\alpha_{H}^{R}=0.3$. We observe that increasing both parameters pushes the shear band toward the center. However, within the parameter range used in our experiments, changing the filling height induces stronger variations in the position of the shear zone compared to varying the applied confining pressure.

In Figure 5, we present the results for the width $\delta / R_{c}$ of the shear band in term of $H / R_{c}$ or $P$. The width of shear band can be considered as the variation of the shear rate around the $R_{s}$ where the shear rate is maximum. This value in our experiments is equal to $\delta$ value obtained from the error function fits to the angular velocity profile. Since the width of the shear band is already narrow, the results of fitting have more pronounced fluctuations compared to other quantities that were presented before. Nevertheless, here we can see that the width of the shear band enhances, on average, with increasing both the pressure and the filling height. Comparing the panels (a) and (b) of Figure 5, one notices that the impact of the filling height is stronger than the external pressure in widening the shear band. We used a simple linear fit to catch the general behavior of $\delta / R_{c}$, to have a quantitative measure of the effect of $H$ and $P$. The slop of the linear fit is equal to $2 \times 10^{-4}$ for $\delta / R_{c}-P$ and 0.2 for for $\delta / R_{c}-H / R_{c}$ plots.

\section{Conclusion}

In summary, we studied the characteristics of shear zones in a split-bottom Couette cell by using the PIV technique. To our knowledge, we are the first to experimentally study the effect of the confining pressure on the evolution of the shear bands. We observed that applying a confining pressure on the granular materials in our experimental geometry has a qualitatively similar effect as increasing the filling height. Our main findings can be outlined as follows: the central location of the shear zone shifts towards the axis of the cell and its width grows as the external pressure or filling height increases. The open shear bands reaching the top surface at low confining pressures or shallow filling heights turn to closed shear zones buried inside the bulk as $H$ or $P$ increases. Moreover, the angular velocity of the particles at the top surface decreases in cases of a larger confining pressure or a higher filling level. We note that the presence of the plexiglass plate may affect the stress transmission in the system as it slightly flattens the top surface and also because of the frictional grain-plate contacts. Our recent numerical results show that the quantities of interest in this study (such as the position and width of the shear zone) are insignificantly affected even under strong stress anisotropies and we have checked that the reported trends in terms of the key parameters remain valid. This is however beyond the scope of the present study. We will compare our empirical findings with the results of numerical simulations elsewhere, to better understanding the mechanisms of shear band formation under the influence of confining pressure.

\section{References}

[1] J. Desrues and G. Viggiani, Int. J. Numer. Anal. Meth. Geomech. 28, 279-321 (2004)

[2] S.B. Savage, Mechanics of granular flows (Springer, Vienna, 467-522, 1993)

[3] R. Moosavi, M.R. Shaebani, M. Maleki, J. Török, D.E. Wolf and W. Losert, Phys. Rev. Lett. 111, 148301 (2013)

[4] C. Xiang et al., Phys. Rev. Lett. 96, 038001 (2006)

[5] S. Luding, Part. Sci. Technol.26, 33-42 (2007)

[6] D. Fenistein and M. van Hecke, Nature 425, 256-256 (2003)

[7] D. Fenistein et al., Phys. Rev. Lett. 92, 094301 (2004)

[8] Y. Forterre and O. Pouliquen, Annu. Rev. Fluid Mech. 40, 1-24 (2008)

[9] D. Fenistein, J. van de Meent and M. van Hecke, Phys. Rev. Lett. 96, 118001 (2006)

[10] T. Unger et al., Phys. Rev. Lett. 92, 214301 (2004)

[11] J.T. Zachary et al., IEEE Trans. Instrum. Meas. 59, 3262-3269 (2010) 